\title{
Methicillin-Resistant Staphylococcus aureus Infections in Patients With Renal Disorders: A Review
}

\author{
Kailash Singha ${ }^{\text {a }}$, Venkata Raju ${ }^{b}$, Ravindra Nikaljic, Sunil Jawale ${ }^{\mathrm{d}}$, \\ Haresh Patel $^{\mathrm{e}}$, Jaishid Ahdal ${ }^{\mathrm{f}, \mathrm{g}}$, Rishi Jain ${ }^{\mathrm{f}}$
}

\begin{abstract}
Methicillin-resistant Staphylococcus aureus (MRSA) infection is a rapidly escalating global health burden. It is not only restricted to patients in the hospital settings but has also rooted deeply in the community settings. With increasing prevalence of life style and kidney diseases, the prevalence of MRSA infections is also expected to rise. MRSA infection plays a major role in renal disorders due to its direct vascular access (VA) thereby making patients undergoing dialysis and renal transplant more vulnerable to infections. Prolonged hospital stay, close proximity to MRSA-infected individual, exposure to broad-spectrum antibiotics, surgery and presence of foreign bodies such as central venous catheters predispose an individual to MRSA infection. Current panel of antibiotic treatment includes vancomycin, teicoplanin, linezolid, daptomycin, tigecycline and ceftaroline. However, emergence of resistant strains and several undesirable features pertaining to safety and tolerability of these drugs have led to limited options available for the management of multidrug-resistant MRSA infection in patients with renal disorders. Therefore, there is an increasing need for developing a new potent antibacterial agent with established renal safety that decreases the mortality and morbidity rates in MRSA-infected renal patients.
\end{abstract}

Keywords: MRSA; Renal disorders; Anti-MRSA agents

\section{Introduction}

Antimicrobial resistance has become a serious public health concern worldwide. In the healthcare and community settings,

Manuscript submitted December 19, 2018, accepted January 22, 2019

andraprastha Apollo Hospitals, New Delhi, India

${ }^{b}$ Raju Institute of Nephrology and Urology, Bangalore, India

${ }^{\mathrm{c}}$ Apollo Hospitals, Navi Mumbai, India

dPoona Hospital and Research Centre, Pune, India

eApollo Hospital, Ahmedabad, India

fWockhardt Ltd, Wockhardt Towers, Bandra Kurla Complex, Mumbai, India gCorresponding Author: Jaishid Ahdal, Wockhardt Ltd, Wockhardt Towers, Bandra Kurla Complex, Mumbai 400051, India.

Email: jahdal@wockhardt.com

doi: https://doi.org/10.14740/wjnu384 the methicillin-resistant Staphylococcus aureus (MRSA) infections have been rising at an alarming rate in the last few years. S. aureus, a gram-positive organism, is an opportunistic pathogen which colonizes on epithelial tissue of skin, skin glands and mucous membrane causing infections ranging from mild (cellulitis) to life-threatening conditions like septic shock. The widespread use of antibiotics (partially due to self-medication and over-the-counter antimicrobials) has resulted in the emergence of resistant strains of $S$. aureus. Resistance to methicillin is determined by mecA gene which expresses for lowaffinity penicillin-binding protein 2 (PBP2) $[1,2]$. MRSA infections are divided into three types: hospital-acquired MRSA (HA-MRSA), community-acquired MRSA (CA-MRSA) and livestock-acquired MRSA (LA-MRSA). Presence of biofilms on biomaterials in the hospitals and other healthcare facilities contributes to the spreading of HA-MRSA among susceptible patient population. Apart from the MRSA infections in healthcare setting, there are CA-MRSA infections arising due to direct or indirect contact with hospitals and care facilities. Key areas of research in renal patients infected with MRSA include genetic factors contributing to the resistance pattern, global epidemiology and its dynamics, the evolution of resistance, and new treatment modalities in patients with renal disorders [3].

\section{Prevalence}

The prevalence of MRSA infections has increased over the years globally. The first case of MRSA was found in 1961. The percentage of invasive MRSA isolates in Europe in 2014 varied from $0.9 \%$ to $56 \%$, with a population-weighted mean of $17.4 \%$. Globally the percentage of $S$. aureus infections lies in the range of $13-74 \%$. In $2005,31.8$ per 100,000 was the rate of invasive MRSA infections and $75 \%$ of these invasive MRSA infections were due to $S$. aureus bacteremia (SAB). According to the national surveillance reports from Asia and Western Pacific Region, S. aureus has been recognized as a very important pathogen, and MRSA incidence was found to be in the range of 2.3-69.1\%. The rate of MRSA bacteremia in Canada, Australia, and Scandinavia has increased in the span of 2000 and $2008(\mathrm{P}=0.035)$ owing to rise in community-acquired infections $(\mathrm{P}=0.013)$ [4]. The study conducted by Suzuki et al showed that MRSA attributed to $26 \%$ of the infections among 42,857 general population in North America and $19.5 \%$ of the infections among 26,613 general population in Europe [5]. 
S. aureus is one of the major reasons for hospital-acquired infections. The highest prevalence rates of HA-MRSA infections $(>50 \%)$ are reported in North and South America, Asia and Malta. Intermediate rates $(25-50 \%)$ are reported in China, Australia, Africa and some European countries, such as Portugal (49\%), Greece (40\%), Italy (37\%) and Romania (34\%). Moreover, Asian countries have been shown to have a very high prevalence rate $(>50 \%)$ of MRSA contributing to hospital-acquired infections such as pneumonia, surgical site infections and bloodstream infections. According to the Asian Network for Surveillance of Resistant Pathogens (ANSORP) study on $S$. aureus in Asia, MRSA accounted for $25.5 \%$ of CAand $67.4 \%$ of HA-MRSA infections [6].

According to the study conducted by Indian Council of Medical Research (ICMR), the prevalence of MRSA in India was $37.3 \%$ in 2017. In this study, the lowest MRSA prevalence was found at All India Institute of Medical Sciences (AIIMS) Delhi $(21 \%)$ while the highest MRSA prevalence was found at Christian Medical College (CMC) Vellore (45\%). This variation in prevalence rate was due to differences in the antibiotic prescription practices followed and the infection control measures employed at different locations. MRSA prevalence at Postgraduate Institute of Medical Education and Research (PGIMER), Chandigarh and Jawaharlal Institute of Postgraduate Medical Education and Research (JIPMER), Puducherry was $43 \%$ and $35 \%$, respectively [7].

About one-third of the general population is colonized with $S$. aureus. Anterior nares are the common site, and other frequent sites are the throat, axilla, rectum, groin, or perineum. The pooled prevalence of MRSA colonization is $1.3 \%(95 \%$ confidence interval (CI): 1.04-1.5\%) globally [5]. For hemodialysis, the percentage of estimated MRSA colonization was 7.2\% (95\% CI: 4.9-9.9\%), while for peritoneal dialysis, it was 1.3\% (95\% CI: $0.5-2.4 \%$ ) [6]. The pooled MRSA prevalence in dialysis patients from European studies was 4.0\% (95\% CI: $1.5-7.7 \%)$, lower than the prevalence in USA 7.9\% $(95 \% \mathrm{CI}$ : 4.4-12.3\%) and Asian countries 10.3\% (95\% CI: 5.7-16.0\%) [8]. A study by Suzuki et al in 2006 reported that MRSA attributed to $38.4 \%$ of infections in 15,618 dialysis patients [5]. Further, Moore et al showed that colonization with MRSA during the peri-operative period can forecast renal allograft failure within 5 years of transplantation independently. The percentage reduction in 1-year, 3-year and 5-year graft survival in MRSA-positive recipients is $100 \%, 86 \%$ and $78 \%$ when compared to $100 \%, 100 \%$ and $93 \%$ respectively in the control group [9].

\section{MRSA in Renal Disorders}

\section{Dialysis}

S. aureus has been reported as the most common infection causing pathogen in dialysis patients due to vascular access (VA) and frequent exposure to hospital environment. Patients undergoing hemodialysis and peritoneal dialysis are more prone to microbial infection [10].

In a study by the US Centres for Disease Control and
Prevention (CDC) in 2005, invasive MRSA infection was observed in approximately 45 out of every 1,000 patients and the risk of infection in hemodialysis patients was observed to be 100 times greater than that in nondialysis patients. Also mortality rate in hemodialysis patients with MRSA infection is five times greater than hemodialysis patients without MRSA infection [10].

Lederer et al performed a study to establish the prevalence of MRSA carriers in out-clinic hemodialysis patients and it was found that end-stage renal disease (ESRD) patients maintained on regular hemodialysis posed a great risk of $S$. aureus infections, owing to exposure to VA site. Nasal MRSA carriage was significantly greater in hemodialysis patients than in hemodialysis personnel (53\% vs. 26\%, P = 0.009) [11].

The study conducted by $\mathrm{Lu}$ et al carried out the surveillance in three groups. The $S$. aureus carriage rate was significantly higher among peritoneal dialysis patients (43.4\%) than hemodialysis patients $(21.8 \%)(\mathrm{P}<0.0001,95 \% \mathrm{CI}: 1.695$ - 4.449). MRSA carriage rates were found to be comparable in both cases. For peritoneal and hemodialysis patients, rates were $2.41 \%(2 / 83)$ and $2.36 \%(12 / 509)$ respectively [12].

\section{Renal transplant}

In solid organ transplantation, the patients are already immunocompromised and therefore, the risk and severity of MRSA infection might increase. Colonization can occur in the pretransplantation or post-transplantation period and is associated with multiple factors consisting of length of surgery, antimicrobial use, length of intensive care unit stay (ICU), usage of drains and/or catheters, and patient comorbidities. In a study done by Cunha et al, it was found that among the 693 renal transplants performed, the MRSA prevalence was $1.3 \%$ [13].

\section{Other renal diseases}

Glomerulonephritis with $S$. aureus infection complicates the disease. Superantigen formation in the bacteria induces host $\mathrm{T}$ cell activation which further increases the interleukins, tumor necrosis factors and immunoglobulin (IgG and IgA) levels in the patients [14].

\section{Causes and Risk Factors for MRSA Infections}

MRSA infections continue to grow in hospital settings and more recently, also in community settings not only in the USA but also across the globe. The increase in the incidence of $S$. aureus infection is partially a consequence of advances in patient care and also due to the pathogen's ability to adapt to changing environment. A growing concern is the emergence of MRSA infections in patients without apparent risk factors. CA-MRSA clones such as ST8 (USA300), ST30, ST59 and ST80 have been spreading rapidly in the community and due to their invading nature, they are contaminating healthcare facilities worldwide really fast. HA-MRSA clones ST239/ 
Table 1. MRSA Risk Groups

\begin{tabular}{l}
\hline Children $<2$ years and adults $\geq 65$ years \\
Athletes \\
Injection drug users \\
Homosexuals \\
Military personnel \\
Inmates of correctional facilities, residential homes or shelters \\
Veterinarians, pet owners and pig farmers \\
Patients with post flu-like illness and/or severe pneumonia \\
Patients with concurrent skin and soft-tissue infections \\
History of colonization or recent infection with CA-MRSA
\end{tabular}

ST241-III-MRSA and ST22-IV-MRSA have been isolated widely $[15,16]$.

Patients who are more susceptible to acquiring MRSA infection are grouped as below in table 1 [15].

Risk factors associated with MRSA infection include prolonged hospital stay, frequent hospitalizations, exposure to broad-spectrum antibiotics, admission to an ICU or burn unit, recent surgery, contact with MRSA-infected patients, presence of foreign bodies such as central venous catheters, presence of comorbidities like diabetes and MRSA colonization. The most common mechanism for introduction of MRSA into an institution is the admission of an infected or colonized patient who serves as a reservoir [17].

\section{Prevention Strategies}

Management of MRSA infection includes proper precautions, prevention and immediate and skilled medical attention. CDC has set up following core interventions to prevent blood stream infection in dialysis patients [5]: 1) To perform monthly hand hygiene observations and share results with the clinical staffs; 2) To follow protocols for the hand hygiene and glove use; 3) To train staff on infection control practices such as access care and aseptic techniques; 4) To evaluate competency of staff every 6 months and upon hire; 5) To perform quarterly VA care observations and assess the aseptic techniques used for connecting and disconnecting catheters and methods for dressing change. Efforts for catheter removal whenever possible should be made (e.g. through patient education) with identifying and considering barriers to permanent VA placement. Catheter hub disinfection should be carried out with use of proper antiseptic after removal and before using of the cap. If closed needleless connector device is used, connector device should be sterilized as per manufacturer's instructions. Antimicrobial ointment should be applied to catheter exit sites during dressing changes; 6) To give standardized education and training on topics such as VA care, hand hygiene, risks related to catheter use, recognizing signs of infection, and instructions for access management to patients when away from the dialysis unit; and 7) To use an alcohol-based chlorhexidine $(>0.5 \%)$ solution as the first-line skin antiseptic agent for central line insertion and during dressing changes. Povidone-iodine (preferably with alcohol) or $70 \%$ alcohol are alternatives for patients with chlorhexidine intolerance [5].

\section{Current Management Choices for MRSA-Infect- ed Renal Patients}

The elementary step in management of MRSA infections in renal patients is to identify and eradicate the source of infection. Catheter, grafts or any other hidden sources which can be a source of infection are detected by magnetic resonance imaging (MRI) or computer tomography (CT) imaging methods [4].

Glycopeptides, a class of antibiotics, are widely used firstline treatment for MRSA infections in renal diseases. This class of antibiotics include drugs such as vancomycin and teicoplanin. They are approved for treatment of complicated skin and skin structure infections, blood stream infections, and infective endocarditis, caused by S. aureus (including MRSA). Their bactericidal action is due to their ability to inhibit bacterial cell wall synthesis. Vancomycin has slow onset of action and is poorly absorbed in some tissues. While US guidelines recommend a fixed dose, European guidelines advise dosing vancomycin based on the trough plasma concentration $\left(\mathrm{C}_{\min }\right)$ to attain ratio of vancomycin area under the curve to minimum inhibitory concentration (MIC) to $\geq 400$ in 24 -h dosing interval. But studies prove that $\mathrm{C}_{\min }$ for $24 \mathrm{~h}$ underestimates the area under the curve by $25 \%$ and therefore, does not give the clear idea of the vancomycin concentration in the body. Patients who achieved $\mathrm{C}_{\min }$ of 15 to $20 \mathrm{mg} / \mathrm{L}$ within $72 \mathrm{~h}$ are very less likely to experience vancomycin failure than patients with $\mathrm{C}_{\min }$ less than $15 \mathrm{mg} / \mathrm{L}$. Teicoplanin has been widely reported to be comparable to vancomycin in efficacy with fewer adverse effects than vancomycin [18]. In a phase II randomized clinical trial, dalbavancin, a semisynthetic derivative of teicoplanin, has been reported to be superior to vancomycin in the treatment of catheter-related bloodstream infections $(87 \% \mathrm{vs}$. $50 \% ; \mathrm{P}<0.05)$ [19]. Daptomycin, a lipopeptide, is an alternative first-line treatment for MRSA infections but is not recommended in pneumonia $[4,19]$.

Televancin and oritavancin are semisynthetic derivatives of vancomycin possessing concentration-dependent bactericidal activity. Mechanism of action of these drugs are either by inhibiting the transglycosylation (polymerization) step of cell wall biosynthesis by binding to the stem peptide of peptidoglycan precursors or by inhibiting the transpeptidation (cross-linking) step of cell wall biosynthesis by binding to the peptide-bridging segments of the cell wall, thereby disrupting bacterial membrane integrity, leading to depolarization, permeabilization, and cell death. Oritavancin has advantage of prolonged half-life and concentration-dependent activity, suitable for single-dose treatment [19].

Oxazolidinones are a group of antibiotics that act against gram-positive bacteria by inhibiting the protein synthesis by binding to the ribosomal 50S subunit. Linezolid and tedizolid are the drugs representing this class. On the basis of several randomized controlled trials, linezolid has been approved for 
Table 2. Limitations of Currently Available Anti-MRSA Antibiotics

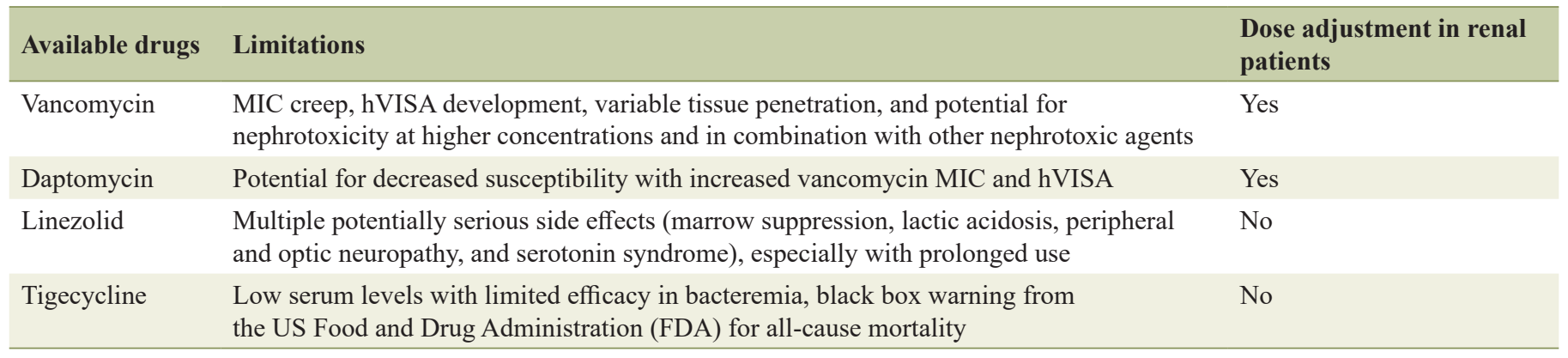

complicated and uncomplicated skin and skin-structure infections and for community-acquired and nosocomial pneumonia in a dose of $600 \mathrm{mg}$ twice daily, intravenously or orally. No dosage adjustment is necessary in chronic kidney disease, but a strict monitoring for adverse effects is recommended [19, 20].

Fifth generation of cephalosporins from the category of $\beta$-lactams are found effective against MRSA infections. Ceftaroline, a broad spectrum cephalosporin, has the ability to bind to PBP2a, an MRSA-specific PBP that has low affinity for most of the other $\beta$-lactam antibiotics. The high binding affinity of ceftaroline to PBP2a correlates well with its low MIC for MRSA [4]. Another class of antibiotics are glycyclines which are derived from tetracyclines and inhibit the bacterial growth by inhibiting the bacterial protein synthesis. Glycyclines are active against resistant organisms like MRSA and one such example is tigecycline which is an effective drug that is in use against MRSA infections. No dosage adjustment is needed in case of renal impairment [20]. Quinupristin/dalfopristin may be used in SAB with reduced susceptibility to vancomycin [4].

Early identification of dialysis patients at risk for complicated SAB can impact patient management in a positive manner. Vandecasteele et al have demonstrated a scoring system based on the presence of four clinical risk factors, for the identification of complicated SAB in hospitalized patients. In this study the patient score is calculated on the basis of points allocated for specific risk factors which include presence of communityacquired $\mathrm{SAB}$, acute systemic infection, persistent fever at $72 \mathrm{~h}$ and follow-up blood culture result at 48 - $96 \mathrm{~h}$ [21].

\section{Limitations and Challenges in Current Manage- ment}

The current available treatments reduce mortality associated with $S$. aureus infections but fail to prevent complications associated with SAB. The resistance to available antibiotic regime has emerged long before and is one of the main reasons behind mortality due to MRSA infections. Therefore, to decrease the bulging infection rate, enforcing preventive measures are necessary. Changes in the epidemiology such as rising prevalence, mortality and morbidity due to MRSA made medical fraternity to look into the limitations of antibiotic regime that is currently followed and the need for new management strategies was developed [21].
The initial antibiotic therapy is often based on observation and experience due to inability to identify and characterize the main cause which can be infectious pathogens. This may sometimes result in inappropriate treatment that can further cause unwanted complications and may lead to increase in hospitalizations and mortality of patients suffering from MRSA. Faster identification of the infection and complications is necessary so appropriate course of antibiotics can be practised and prevents the infection growing to the necrotic stage. Diagnostic techniques with fast and accurate method for identification of infection should be invented and adapted so that targeted antibiotic therapy could be given [21].

Further, there are studies that report the emergence of resistance against newly discovered antibiotics. In Australia as many as $17 \%$ of MRSA isolates were found to be resistant to the newly invented drug ceftaroline. Increasing use of vancomycin has been associated with the emergence of vancomycinintermediate $S$. aureus (VISA) and heteroresistant VISA (hVISA) isolates, causing reduced susceptibility to glycopeptide antibiotics. Developed resistance to vancomycin, daptomycin and linezolid, identifying interactions between HA-, CA- and LA-forms of MRSA, contaminating new reservoirs, and lastly, monitoring interactions between community setting and hospital infections are some of the current challenges [22].

Currently available antibiotics have multiple limitations when used as monotherapy against MRSA. Vancomycin has bactericidal activity against MRSA infections depending on strain-specific factors. Some of the drawbacks of vancomycin monotherapy are slow increase in MIC of MRSA over time (MIC creep), increase in $S$. aureus heteroresistance to vancomycin and pharmacokinetic and pharmacodynamic factors affecting drug delivery to the expected site. Daptomycin, possessing similar activity like vancomycin, is inactivated by lung surfactants, and therefore is ineffective in pneumonia. Linezolid is not used as front-line treatment owing to its bacteriostatic nature and for prolonged use due to side effects listed in table 2. Tigecycline which is highly protein bound has low serum levels during bacteremia, making it ineffective [23]. The limitations and dose adjustment of currently available anti-MRSA antibiotics are summarized in Table 2 [20, 24, 25].

The identification and synthesis of new drugs with novel modes of action that may circumvent the developed antibiotic resistance for existing antibiotics are challenging. But to overcome the increasing incidence and mortality in cases of MRSA, discovery of new drugs has become a necessity [21]. 


\section{Evolving Trends}

The need for newer management techniques has become essential after continuous development of resistant strains against the established MRSA antibiotic therapies. Early identification of bloodstream infections, combined with the rapid initiation of appropriate antibiotic therapy, is likely to reduce the risk of complications. Development of new chemical entities or derivatizing the existing drug entities may prove beneficial than existing drug molecules against MRSA infections in renal diseases [21].

Combination therapy of antibiotics which is widely used in clinical practice (e.g. a glycopeptide or daptomycin with a $\beta$-lactam antibiotic) is being explored as a novel strategy in the fight against MRSA. Combination therapies like vancomycin and nafcillin, ceftabiprole and vancomycin, ceftabiprole and daptomycin, and daptomycin and rifampicin are studied in regards to MRSA endocarditis and acute bacterial skin and skin structure infections (ABSSSIs). Bal et al demonstrated that complete eradication of MRSA bacteremia was achieved in patients with bloodstream infections by combination therapy of vancomycin and a $\beta$-lactam antibiotic rather than vancomycin monotherapy [22].

Ceftabiprole, a fifth-generation cephalosporin, is found to be more active than ceftaroline. Its broad spectrum activity is observed against MRSA, VISA and daptomycin. A multicentre retrospective observational study demonstrated a $68 \%$ success rate for ceftaroline (with or without combination therapy) among 211 patients with MRSA bacteremia from 2011 to 2015 $[22,26]$.

Newly discovered lipoglycopeptides like dalbavancin, televancin, and oritavancin are licensed by US FDA currently against ABSSSIs and hospital-associated pneumonia (HAP). Their use can further be extended in the treatment of bacteremia, infective endocarditis, and osteomyelitis in the future [22].

Delafloxacin approved by FDA in June 2017 is a dualtargeting fluoroquinolone which forms breakable complexes with DNA and topoisomerase IV or DNA gyrase and inhibits the activity of these enzymes in both gram-positive and gramnegative bacteria respectively. Delafloxacin has been observed to show potent activity against MRSA where MICs are higher $\left(\mathrm{MIC}_{50} 0.25 \mu \mathrm{g} / \mathrm{mL}\right.$ ) for fluoroquinolone-resistant isolates as compared to fluoroquinolone-susceptible isolates $\left(\mathrm{MIC}_{50}\right.$ $0.004 \mu \mathrm{g} / \mathrm{mL}$ ). Similar to delafloxacin, zabofloxacin is also extremely potent against gram-positive bacteria associated with respiratory tract infections such as $S$. pneumoniae $\left(\mathrm{MIC}_{50}\right.$ for MRSA is $2 \mu \mathrm{g} / \mathrm{mL}$ as compared to $0.125 \mu \mathrm{g} / \mathrm{mL}$ for delafloxacin) $[22,27]$. Other fluoroquinolones including WCK2349 and WCK771 subclasses of benzoquinolizine fluoroquinolone levonadifloxacin (in phase III trial by Wockhardt) [28] and nemonoxacin (phase II) [29] are new chemical entities that are currently undergoing clinical trials. Fluoroquinolones, such as norfloxacin, ciprofloxacin, ofloxacin and levofloxacin, target staphylococcal topoisomerase IV causing widespread cross-resistance among members of this class due to the relative mutational vulnerability of topoisomerase IV. However, WCK 771 was found more potent than moxifloxacin, trovafloxacin, levo- floxacin, and ciprofloxacin with potency comparable to that of clinafloxacin. The strong potency of WCK 771 against MSSA and MRSA strains is due to its unique bactericidal mechanism of action wherein it targets DNA gyrase (topoisomerase II). However, WCK 771 is not a substrate of the NorA pump so MIC of drug is not related to reserpine. These factors create high intracellular drug concentrations even in the resistant strains ultimately leading to bacterial lysis. WCK 771 and clinafloxacin have been found to have a stronger potency against VISA strains (MICs $=1 \mu \mathrm{g} / \mathrm{mL})$ [30].

\section{Conclusions}

Staphylococcus, a ubiquitous microorganism, can survive under extreme conditions and colonize in patients with renal disorders without any symptoms. Current management strategies for the treatment of renal disorders with staphylococcus infections are becoming increasingly limited due to the rising incidence of resistant pathogens such as MRSA. Monotherapies like vancomycin, teicoplanin, daptomycin or linezolid are the first-line treatments against MRSA in renal disorders but there are limitations for these drugs in terms of development of resistance and side effects. To prevent resistance to monotherapy, combination therapy of available antibiotics is being explored. But combination therapies have their own disadvantages in terms of drug-drug interaction, toxicity and the risk of increasing multidrug-resistant bacteria. Hence, there is an urgent need to develop new antimicrobial agents with established renal safety to combat increasing multidrug-resistant MRSA infections, thereby decreasing the associated mortality and morbidity in infected renal patients.

\section{Conflict of Interest}

Authors Kailash Singh, Venkata Raju, Ravindra Nikalji, Sunil Jawale and Haresh Patel have nothing to declare. Jaishid Ahdal and Rishi Jain are employees of Wockhardt Ltd. Mumbai, India.

\section{References}

1. Shorr AF. Epidemiology and economic impact of meticillin-resistant Staphylococcus aureus: review and analysis of the literature. Pharmacoeconomics. 2007;25(9):751768.

2. Nathwani D, Morgan M, Masterton RG, Dryden M, Cookson BD, French G, Lewis D, et al. Guidelines for UK practice for the diagnosis and management of methicillin-resistant Staphylococcus aureus (MRSA) infections presenting in the community. J Antimicrob Chemother. 2008;61(5):976-994.

3. Grema H, Geidam Y, Gadzama G, Ameh J, Suleiman A. Methicillin Resistant Staphylococcus aureus (MRSA): A Review. Advances in Animal and Veterinary Sciences. 2015;(3):79-86. 
4. Hassoun A, Linden PK, Friedman B. Incidence, prevalence, and management of MRSA bacteremia across patient populations-a review of recent developments in MRSA management and treatment. Crit Care. 2017;21(1):211.

5. Suzuki M, Satoh N, Nakamura M, Horita S, Seki G, Moriya K. Bacteremia in hemodialysis patients. World J Nephrol. 2016;5(6):489-496.

6. Kang CI, Song JH. Antimicrobial resistance in Asia: current epidemiology and clinical implications. Infect Chemother. 2013;45(1):22-31.

7. Rajkumar S, Sistla S, Manoharan M, Sugumar M, Nagasundaram N, Parija SC, Ray P, et al. Prevalence and genetic mechanisms of antimicrobial resistance in Staphylococcus species: A multicentre report of the indian council of medical research antimicrobial resistance surveillance network. Indian J Med Microbiol. 2017;35(1):53-60.

8. Zacharioudakis IM, Zervou FN, Ziakas PD, Mylonakis E. Meta-analysis of methicillin-resistant Staphylococcus aureus colonization and risk of infection in dialysis patients. J Am Soc Nephrol. 2014;25(9):2131-2141.

9. Moore C, Davis NF, Burke JP, Power R, Mohan P, Hickey D, Smyth G, et al. Colonisation with methicillin-resistant Staphylococcus aureus prior to renal transplantation is associated with long-term renal allograft failure. Transpl Int. 2014;27(9):926-930.

10. Centers for Disease Control. Invasive methicillin-resistant Staphylococcus aureus infections among dialysis patients - United States, 2005. MMWR Morb Mortal Wkly Rep. 2007;56(9):197-199.

11. Lederer SR, Riedelsdorf G, Schiffl H. Nasal carriage of meticillin resistant Staphylococcus aureus: the prevalence, patients at risk and the effect of elimination on outcomes among outclinic haemodialysis patients. Eur J Med Res. 2007;12(7):284-288.

12. Lu PL, Tsai JC, Chiu YW, Chang FY, Chen YW, Hsiao CF, Siu LK. Methicillin-resistant Staphylococcus aureus carriage, infection and transmission in dialysis patients, healthcare workers and their family members. Nephrol Dial Transplant. 2008;23(5):1659-1665.

13. Oliveira-Cunha M, Bowman V, di Benedetto G, MituPretorian MO, Armstrong S, Forgacs B, Tavakoli A, et al. Outcomes of methicillin-resistant Staphylococcus aureus infection after kidney and/or pancreas transplantation. Transplant Proc. 2013;45(6):2207-2210.

14. Zeledon JI, McKelvey RL, Servilla KS, Hofinger D, Konstantinov KN, Kellie S, Sun Y, et al. Glomerulonephritis causing acute renal failure during the course of bacterial infections. Histological varieties, potential pathogenetic pathways and treatment. Int Urol Nephrol. 2008;40(2):461-470.

15. Boucher HW, Corey GR. Epidemiology of methicillin-resistant Staphylococcus aureus. Clin Infect Dis. 2008;46(Suppl 5):S344-349.

16. Stefani S, Chung DR, Lindsay JA, Friedrich AW, Kearns AM, Westh H, Mackenzie FM. Meticillin-resistant Staphylococcus aureus (MRSA): global epidemiology and harmonisation of typing methods. Int J Antimicrob Agents. 2012;39(4):273-282.
17. Mulligan ME, Murray-Leisure KA, Ribner BS, Standiford HC, John JF, Korvick JA, Kauffman CA, et al. Methicillin-resistant Staphylococcus aureus: a consensus review of the microbiology, pathogenesis, and epidemiology with implications for prevention and management. Am J Med. 1993;94(3):313-328.

18. Lee CH, Tsai CY, Li CC, Chien CC, Liu JW. Teicoplanin therapy for MRSA bacteraemia: a retrospective study emphasizing the importance of maintenance dosing in improving clinical outcomes. J Antimicrob Chemother. 2015;70(1):257-263.

19. Boswihi S, Udo E. Methicillin-resistant Staphylococcus aureus: An update on the epidemiology, treatment options and infection control. Current Medicine Research and Practice. 2018;(8):18-24.

20. Vandecasteele SJ, Boelaert JR, De Vriese AS. Staphylococcus aureus infections in hemodialysis: what a nephrologist should know. Clin J Am Soc Nephrol. 2009;4(8):1388-1400.

21. Naber CK. Future strategies for treating Staphylococcus aureus bloodstream infections. Clin Microbiol Infect. 2008;14(Suppl 2):26-34.

22. Bal AM, David MZ, Garau J, Gottlieb T, Mazzei T, Scaglione F, Tattevin P, et al. Future trends in the treatment of methicillin-resistant Staphylococcus aureus (MRSA) infection: An in-depth review of newer antibiotics active against an enduring pathogen. J Glob Antimicrob Resist. 2017;10:295-303.

23. Tarai B, Das P, Kumar D. Recurrent Challenges for Clinicians: Emergence of methicillin-resistant staphylococcus aureus, vancomycin resistance, and current treatment options. J Lab Physicians. 2013;5(2):71-78.

24. Nguyen HM, Graber CJ. Limitations of antibiotic options for invasive infections caused by methicillin-resistant Staphylococcus aureus: is combination therapy the answer? J Antimicrob Chemother. 2010;65(1):24-36.

25. Dixit D, Madduri RP, Sharma R. The role of tigecycline in the treatment of infections in light of the new black box warning. Expert Rev Anti Infect Ther. 2014;12(4):397400.

26. Zasowski EJ, Trinh TD, Claeys KC, Casapao AM, Sabagha N, Lagnf AM, Klinker KP, et al. Multicenter observational study of ceftaroline fosamil for methicillinresistant staphylococcus aureus bloodstream infections. Antimicrob Agents Chemother. 2017;61(2)

27. Van Bambeke F. Delafloxacin, a non-zwitterionic fluoroquinolone in Phase III of clinical development: evaluation of its pharmacology, pharmacokinetics, pharmacodynamics and clinical efficacy. Future Microbiol. 2015;10(7):1111-1123.

28. Cited on http://wockhardtdiscovery.com/wck-771-i-v/. on 17 Aug 2018.

29. Poole RM. Nemonoxacin: first global approval. Drugs. 2014;74(12):1445-1453.

30. Patel MV, De Souza NJ, Gupte SV, Jafri MA, Bhagwat SS, Chugh Y, Khorakiwala HF, et al. Antistaphylococcal activity of WCK 771, a tricyclic fluoroquinolone, in animal infection models. Antimicrob Agents Chemother. 2004;48(12):4754-4761. 